\title{
MISFIT AND LATTICE PARAMETERS OF SINGLE CRYSTAL AM1 SUPERALLOY: \\ EFFECTS OF 'TEMPERA'TURE, PRECIPITATE MORPHOLOGY AND $\gamma-\gamma$, INTERFACIAL STRESSES
}

\author{
A. Royer and P. Bastie \\ Laboratoire de spectrométrie physique \\ BP 87, 38402 St Martin d'Hères cedex, France
}

\begin{abstract}
:
The lattice mismatch between the $\gamma$ and $\gamma^{\prime}$ phases and the tetragonal distortion of the lattice cells of single crystal specimens of the nickel-based superalloy AMI have been measured respectively by high resolution neutron diffraction and $\gamma$-ray diffraction techniques. Both temperature evolution on heating and time evolution during annealing of the diffraction profiles were analysed. The misfit depends on the precipitates morphology and is not an intrinsic property of the material. Tetragonal distortions of the $\gamma$ phase have been evidenced and are mainly related to internal stresses at the $\gamma-\gamma^{\prime}$ interfaces. A transition from an elastic accommodation to a plastic relaxation was experimentally observed it the $\gamma-\gamma$ ' interfaces. To interpret the temperature evolution of the misfit and of the distortion of the cubic cells, a model is proposed for the evolution of the lattice parameters of the two phases. Similar description explains the temperature behaviour of the lattice parameters of the CMSX-2 superalloy showing that $\gamma-\gamma^{\prime}$ lattice mismatch is negative at room temperature.
\end{abstract}

Introduction :

The value and the sign of the lattice parameter mismatch $\delta$ between the $\gamma$ and $\gamma^{\prime}$ phases of single crystal superalloys are often considered as comparison criteria between superalloy species. Their influence on the directional coarsening under stress at high temperature is very important as the shape and the size of the precipitates are strongly dependent on the misfit $[1,2]$. However the determination of the lattice parameter mismatch usually defined as $\delta=2\left(a_{\gamma^{\prime}}-a_{\gamma}\right) /\left(a_{\gamma}+a^{\prime}\right)$, has been the subject of numerous controversies and its measurement at high temperature (the using temperature of these alloys) is always an open and difficult question $[3,4,5,6,7]$. This is due to the facts that :

- $\delta$ is small $\left(\delta \approx 10^{-3}\right)$

- samples present segregation and chemical inhomogeneities even after standard heat treatments

- single crystals are not perfect (typical mosaicity of about $30 \mathrm{~min}$ of arc or more) $[8,9]$

- internal stresses at the interfaces between matrix and precipitates modify the lattice parameters and distort the lattice cell. It is important to notice that difference must be done between lattice parameters of isolated phases and of the real biphasic materials [10]. Furthermore tetragonal distortion has been also reported for some superalloys $[11,12,13]$.

In order to detcrmine the origin of these controversics, systematic study of temperature dependence of the misfit and of the tetragonal distortion was undertaken. The influence of the thermomechanical history of the sample was also analysed. In situ experiments were performed in bulk samples using neutron and $\gamma$-ray radiations in order to avoid perturbation related to quenching, to oxidation or surface relaxation and also in order to average over dendritic and interdendritic regions.

\section{Experimental procedure:}

High resolution neutron diffraction experiments were carried out for determination of the lattice parameter distribution using a two nearly parallel crystal arrangement. This set up allows to minimize the instrumental contribution to the broadening of the diffraction peaks [14] and it becomes possible to operate at large Bragg angle $\left(\approx 80^{\circ}\right)$ in order to increase the sensitivity. Fxperiments were carried out on the Institut Laue Langevin (ILL, France) facility S21. Special cares and detailed procedure used in analysing the superalloy data are given in [15]. More details concerning the experimental conditions of the measurements reported in this paper are given in [7].

$\gamma$-ray diffractometry is usually used to measure the mosaicity of the sample [16]. In the particular case of single crystal superalloys a special feature occurs related to the cuboidal morphology of the precipitates. The full width at half maximum (FWHM) of the reflections (200) and (220) should have the same value if the lattice cells are cubic. The measured values are different and reflect a 
tetragonal distortion of the cells, the tetragonal axis being equivalently distributed along the three $<\mathrm{h} 00>$ axes. The principle of these non standard measurements is fully described in [13]. The value of the tetragonal distortion is related to the difference $2 \varepsilon=$ FWHM(220) - FWHM(200). In the present study measurements were performed on the $\gamma$-ray diffractometer of ILL with a radioactive gold source $(\lambda=0.03 \AA)$.

\section{Materials:}

This paper focuses on AMI single crystal superalloy. Similar studies have been done on CMSX-2 and are in agreement with results reported below. The composition of these two superalloys are given table $\mathbf{I}$.

Table I: Chemical composition (in weight \%) of AM1 and CMSX-2 superalloys.

\begin{tabular}{|l|l|l|l|l|l|l|l|l|}
\hline element & $\mathrm{Ni}$ & $\mathrm{Co}$ & $\mathrm{Cr}$ & $\mathrm{Mo}$ & $\mathrm{W}$ & $\mathrm{Al}$ & $\mathrm{Ti}$ & $\mathrm{Ta}$ \\
\hline AMl & balance & 6.5 & 7.5 & 2.0 & 5.5 & 5.3 & 1.2 & 8.0 \\
\hline CMSX-2 & balance & 4.6 & 8.0 & 0.6 & 7.9 & 5.6 & 0.9 & 5.8 \\
\hline
\end{tabular}

Two different types of samples were studied:

- crept sample $\left(140 \mathrm{MPa}, 1050^{\circ} \mathrm{C}, \varepsilon=0.58 \%\right)$ with rafted precipitates

- reference samples cut in an "as cast" bar. Samples selected $(\emptyset=3 \mathrm{~mm}, h=10 \mathrm{~mm}$ for $\gamma$-ray diffraction experiment and $10 * 15 * 3 \mathrm{~mm}^{3}$ for neutron experiments) were chosen for their low mosaicity (typically $10 \mathrm{~min}$ of arc).

Measurements were performed

- on the crept sample for increasing temperature from room temperature up to $1300^{\circ} \mathrm{C}$ (temperature of complete solutionizing of the $\gamma^{\prime}$ phase), and then for decreasing temperature. Two different directions were analysed, parallel and perpendicular to the rafting plane.

- on reference samples with different initial thermal history

$$
\begin{aligned}
& \text { - as cast } \\
& \text { - homogenized }\left(1300^{\circ} \mathrm{C} / 30 \mathrm{~min}\right) \\
& \text { - heat treated (as homogenized }+1050^{\circ} \mathrm{C} / 16 \mathrm{~h} \text { ) }
\end{aligned}
$$

for increasing and decreasing temperature and during annealing at chosen temperatures between $1000^{\circ} \mathrm{C}$ and $1250^{\circ} \mathrm{C}$ after a complete solutionizing of the $\gamma^{\prime}$ phase $\left(1300^{\circ} \mathrm{C} / 30 \mathrm{~min}\right)$.

$\gamma$-ray and neutron rocking curves were recorded in less than 20 minutes. This duration is short enough compared with the microstructure evolution of the superalloy.

Results:

Measurements at room temperature on the crept sample reveal a first surprise. The value of the misfit depends on the crystallographic direction. Its value is $+3.310^{-3}$ along the $<002>$ axis perpendicular to the rafts and is $-1.410^{-3}$ along the $\left.<200\right\rangle$ and $\left.<020\right\rangle$ axis parallel to the rafts. For the reference sample the misfit value is the same for the three $\langle$ hol $>$ directions and is negative, close to zero in the case of the homogenized sample. These facts are sufficient to explain the controversies about the misfit values given in the literature. The value and even the sign of the misfit depend on the thermomechanical history of the sample. The same measurements were performed in temperature up to complete solutionizing of the $\gamma$ ' phase. Figure 1 reports the results for the crept sample (a) and the homogenized reference one (b). A detailed analysis of these results is given in [7].

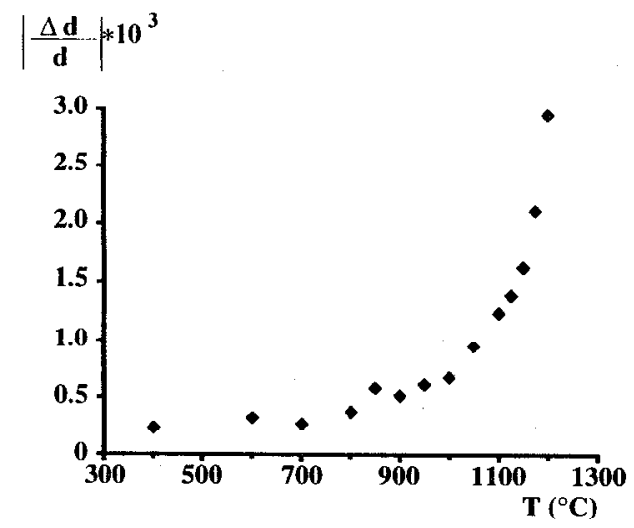

(b)

Figure 1: Temperature dependence of (a) lattice misinatch for the crept sample parallel (200) and perpendicular (002) to the rafts and (b) difference between the largest and the smallest lattice parameters for the homogenized reference sample 
The most striking observation concerns the temperature behaviour of the misfit. It is hugely dependent on the morphology of $\gamma$ ' precipitates and on the crystallographic direction analysed. However we have shown that a crystallographic quantity almost independent of the history of the sample can be found in considering the value of the misfit averaged over the three cube directions:

$$
<\delta>=1 / 3\left(\delta_{200}+\delta_{020}+\delta_{002}\right) \approx 1 / 3 \Delta V / V
$$

where $\Delta V / V$ is the relative change in unit cell volume between the two phases.

After solutionizing of the $\gamma^{\prime}$ phase at high temperature and precipitation, the anisotropy of the diffraction pattern for the crept sample is lost; it "has forgotten" its mechanical and thermal history, from the lattice parameter point of view.

$\gamma$-ray diffraction measurements were performed in the same temperature range. The FWHM of the (200) and (220) reflections are reported in figure 2 for the homogenized reference sample.
Below $900^{\circ} \mathrm{C}$ a constant difference $2 \varepsilon$ of about 1 minute of arc is observed. For higher temperature this difference increases, reaches a maximum close to 3 minutes of arc at $1150^{\circ} \mathrm{C}$ and then decreases and disappears with the complete solutionizing of the $\gamma^{\prime}$ phase.

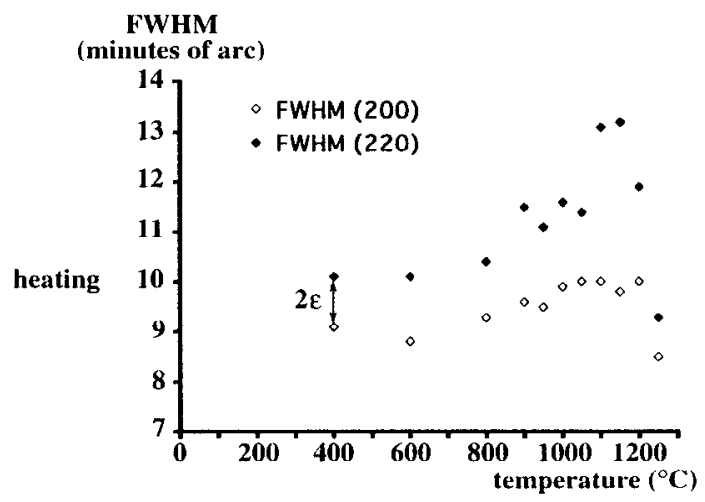

Figure 2: Behaviour versus temperature of the FWHM of the $\gamma-1$ ay rocking curves for the (200) and (220) rellections in the case of the homogenized reference sample

annealing time

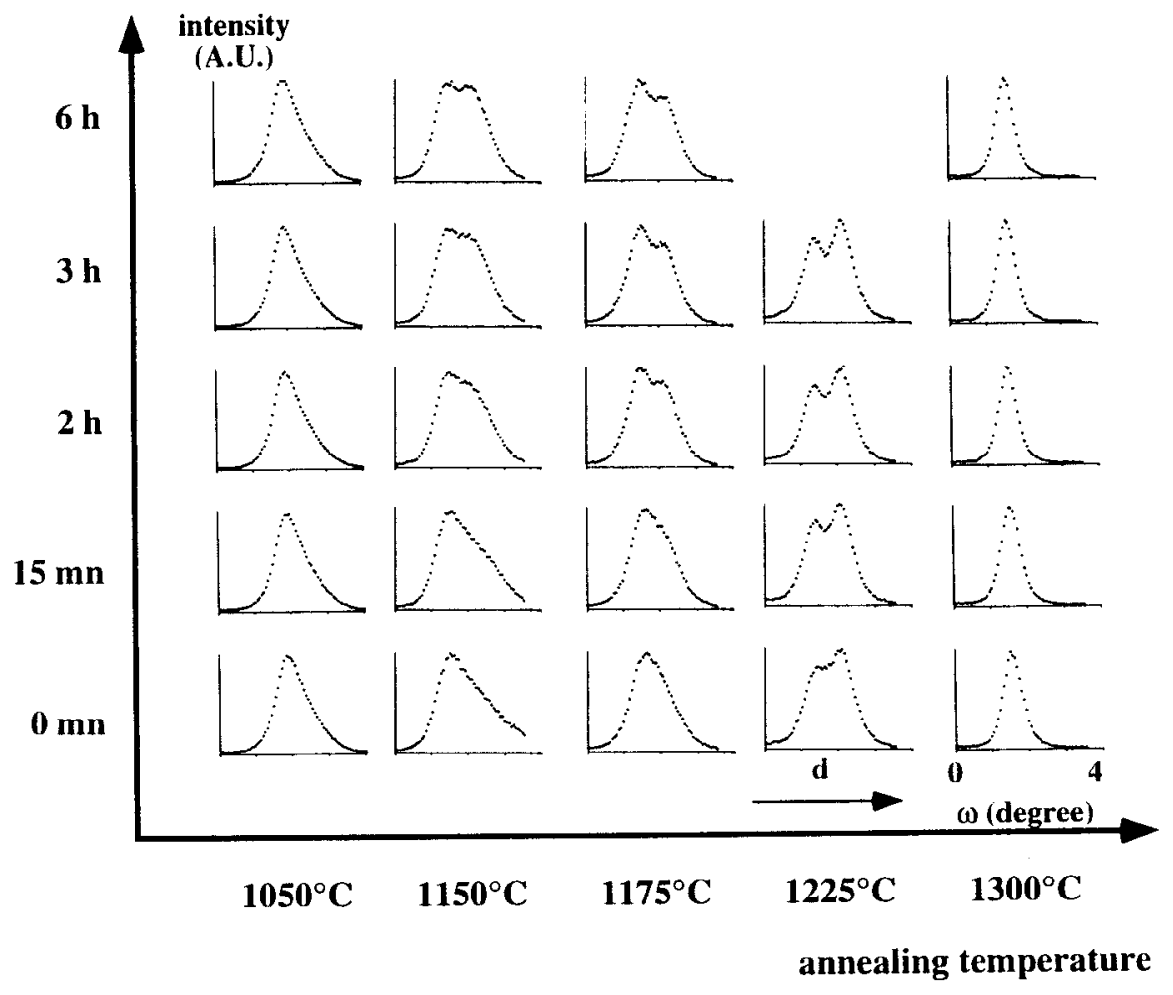

Figure 3: Time evolution of the neutron profile for annealing at different temperature 
The decreasing of the $2 \varepsilon$ value also occurs at $1050^{\circ} \mathrm{C}$ but much slower as seen in figure 5 . At this temperature the characteristic time would be of the order of few hundred hours. These observations show that the lattice parameter spreading and the tetragonal distortion are related to internal stresses due to the $\gamma-\gamma^{\prime}$ phase interfaccs. During annealing a relaxation of these internal stresses occurs. Each phase tends to recover its cubic structure; the coherency of the interfaces disappears progressively.

\section{Discussion and interpretation:}

Due to the internal stresses at the $\gamma-\gamma^{\prime}$ interface, induced by the negative lattice mismatch, the cuboidal precipitates are triaxially expanded while the $\gamma$ corridors are rather biaxially compressed due to their platelet shape. So in a first approximation, one can assume that the unit cell of the $\gamma^{\prime}$ phase remains cubic and that of the $\gamma$ phase becomes tetragonal explaining the distortion observed by $\gamma$-ray diffractometry at room temperature. On heating, the volume of the unit cell of the $\gamma$ phase increases faster than that of the $\gamma$ ' phase [7]; so the internal stresses increase too. The lattice coherency is maintained at the interface until a threshold temperature, between $900^{\circ} \mathrm{C}$ and $1000^{\circ} \mathrm{C}$ and no significant change is observed both on the diffraction profile and for the $2 \varepsilon$ values. Above this threshold, the elastic accommodation is progressively replaced by a plastic relaxation provided by incoming dislocations in an attempt to reduce interfacial stresses. As shown by the annealing measurements the relaxation rate depends strongly on the temperature. So in fact when the rate is slow, this plastic relaxation does not occur simultaneously on the six faces of each precipitate but sequentially and at random. This explains the rapid increase of both $|\Delta d / d|$ and the tetragonal distortion observed during the analysis of increasing temperature up to $1150^{\circ} \mathrm{C}$, because some interfaces are relaxed and some others not. This relaxation becomes faster and faster when the annealing temperature becomes higher. This explains why above $1150^{\circ} \mathrm{C}$ the diffraction profiles show two well split peaks since the beginning of the annealing and why the cell distortion disappears as relaxation becomes rapidly complete in all directions. The two phases recover their cubic structure.

From these observations it becomes possible to explain the temperature behaviour of the misfit parameter on reference sample during heating. Figure 6 gives a schematic description of this qualitative model. It is based on a transition from purely elastic accommodation to a progressively plastic relaxation provided by the development of regular dislocation networks at the matrixprecipitate interfaces. For the clarity of the presentation, we assume that $\gamma$ ' phase kept always its cubic structure. This is not exactly true, in particular when only some interfaces are relaxed, but the major tetragonal distortion occurs in the $\gamma$ phase. Furthermore it remains possible to add the $\gamma$ ' phase distortion for a more quantitative analysis of the neutron and $\gamma$-ray diffraction profiles.
On figure 6 (a) the temperature evolution of the lattice parameters of the $\gamma$ and $\gamma^{\prime}$ phase is schematized. At low temperature (below $900^{\circ} \mathrm{C}$ ) the interfaces are coherent so the $\gamma$ phase is biaxially compressed by the bounding to the $\gamma$ ' phase. The two lattice parameters of the $\gamma$ phase parallel to the interface are close to those of the $\gamma^{\prime}$ phase (a $\gamma$-). The third one expands because of the Poisson coefficient (a $\gamma+$ ). The cell of the $\gamma$ phase is tetragonal with a c/a ratio larger than one (figure 6 (b1)). Between $900^{\circ} \mathrm{C}$ and $1150^{\circ} \mathrm{C}$ some of the interfaces become partially incoherent by plastic relaxation along one of the two stressed directions. The unit cell of the $\gamma$ phase remains tetragonal but now only one direction is compressed and the c/a ratio becomes smaller than one (figure 6 (b2)). Both the $\Delta d / d$ and the tetragonal distortion increase. Above $1150^{\circ} \mathrm{C}$ the plastic relaxation is complete, the unit cells of the $\gamma$ and $\gamma$ ' phases recover their cubic structure. The tetragonal distortion disappears while $\Delta \mathrm{d} / \mathrm{d}$ continue to increase (figure 6 (b3)). This interpretation is conforted by the observations made during the annealings. The progressive vanishing of the tetragonal distortion and the better resolution of the two peaks in the neutron diffraction profiles are in accordance with the plastic relaxation of interfacial stresses. Below $1150^{\circ} \mathrm{C}$ the relaxation time is large and only a partial relaxation is observed. This fact is due to the slowness of the process. For a given interface only one direction is relaxed (figure 6 (b)) leading to a large tetragonal distortion which decreases with annealing time. This temperature evolution of the lattice parameters explains why it is not possible to attribute a priori one diffraction pcak to one phase.

Several other observations are consistent with this description. Indeed, the existence of partial relaxation at intermediate temperatures is compatible with the observation, after long time annealing, of periodic dislocation networks on some of the interfaces [18]. This may also explain the occurrence of the "bamboo" texture reported in the CMSX-2 after a heat treatment of $1000 \mathrm{~h}$ at $1000^{\circ} \mathrm{C}[12]$, the oriented coalescence of the precipitates being favoured by the presence, locally, of a particular set of dislocations $[19,20]$.

\section{Conclusion:}

From these high resolution neutron diffraction and $\gamma$-ray diffraction measurements on reference and crept samples, the following remarks and conclusions can be drawn:

- The thermomechanical history and then the morphology of the $\gamma^{\prime}$ precipitates is an important parameter to be taken into account for measurements of lattice parameter mismatch. Mismatch measured only along one crystallographic direction does not provide intrinsic characteristic for a particular family of superalloy. But the "average" misfit for the three $<\mathrm{h} 00>$ directions is almost independent of the morphology of precipitates and then can be 
chosen as a relevant parameter for a structural characterisation. For alloy AM1, it is close to zero at room temperature (slightly negative) and becomes negative on heating due to the larger thermal expansion of the $\gamma$ phase compared to that of the $\gamma$ ' phase.

- A qualitative model explaining the temperature behaviour of the lattice parameters during heating and annealing is given. The transition from purely elastic accommodation to a progressively plastic relaxation provided by the development of regular dislocation networks at the matrix-precipitate interfaces has been demonstrated. The previously observed distortion of the cubic unit cells and the shape of neutron diffraction profiles are correctly interpreted by this model which is coherent with previous electron microscopy observations.

\section{Annex:}

Similar experiments made on the CMSX-2 single crystal superalloy have shown that the lattice parameters have the same behaviours as those of the AM1 superalloy. The same mechanism is involved for the stress relaxation. The average misfit measured is negative from room temperature to the temperature of the complete $\gamma$ ' phase solutionizing. Its value is $-1.410^{-3}$ at room temperature and decreases to $-3.010^{-3}$ at $1200^{\circ} \mathrm{C}$. In opposition to the usually admitted misfit sign at room temperature, the measured value is negative and then there is no inversion of the misfit sign around $800^{\circ} \mathrm{C}$. Properties of the material in this temperature range cannot be related to a null value of the misfit. This negative value at room temperature suggested in [7] has been confirmed by recent X-ray measurements performed on a Philips MRD diffractometer [21]

\section{References:}

1. T.M. Pollock and A.S. Argon, "Directional Coarsening in Nickel-Based Single Crystals with High Volume Fractions of Coherent Precipitates", Acta Metall. Mater. 42 (1994), 1859-1874

2. M. Veron, Y. Brechet and F. Louchet, "Directional Coarsening of Nickel Based Superalloys: Computer Simulation at Mesoscopic Scale", Acta Metall. Mater. (1996), in press

3. D.F. Lahrman et al., "Investigation of Techniques for Measuring Lattice Mismatch in a Rhenium Containing Nickel Base Superalloy", Acta Metall., 36 (1988), 1309-1320

4. H-A. Kuhn et al., "An X-Ray Study of Creep-Deformation Induced Changes of the Lattice Mismatch in the $\gamma^{\prime}$-Hardened Monocrystalline Nickel-Base Superalloy SRR 99", Acta Metall., 39 (1991), 2783-2794

5. U. Glatzel and A. Muller, "Neutron Scattering Experiments with a Nickel Basc Superalloy. Part I: Material and Experiment", Scripta Metall. Mater. 31 (1994), 285-290.
6. U. Glatzel, "Neutron Scattering Experiments with a Nickel Base Superalloy. Part II: Analysis of Intensity Profiles", Scripta Metall. Mater.. 31 (1994), 291-296

7. A. Royer et al., "Temperature Dependence of the Lattice Mismatch of the AM1 Superalloy. Influence of the $\gamma$ ' Precipitates Morphology", Phil. Mag. A. 72 (1995), 669-689,

8. D. Bellet, P. Bastie and J. Baruchel, "White Beam Synchrotron Topography and $\gamma$-ray Diffractometry Characterisation of the Crystalline Quality of Single Grain Superalloys: Influence of the Solidification Conditions", J. Phys. D: Appl. Phys., 26 (1993), A50-A52

9. N. Siredey et al., "Dendritic Growth and Crystalline Quality of Nickel-Base Single Grains", J. Crystal Growth _ 130 (1993), 132146

10. D. Grose and G. Ansell, "The Influence of Coherency Strain on the Elevated Temperature Tensile Behaviour of $\mathrm{Ni}-15 \mathrm{Cr}-\mathrm{Al}-\mathrm{Ti}$ Mo Alloys", Met. Trans. A, 12 (1981), 1631-1645

11. A.J. Porter et al., "The application of Convergent-Beam Electron Diffraction to the Detection of Small Symmetry Changes Accompanying Phase Transformations, part II: Recrystallization of Superalloy", Phil. Mag. A, 44 (1981), 1135-1148

12. R. Bonnet and A. Ati, "Mise en Evidence par MET d'une Phase Ordonnée Légèrement Quadratique dans le Superalliage CMSX-2 Recuit", J.Microsc. Spectrosc. Electron., 14 (1989), 169-180,

13. D. Bellet and P. Bastie, "Temperature Dependence of the Lattice Parameter of the $\gamma$ and $\gamma^{\prime}$ Phases in the Nickel-Based Superalloy CMSX-2, Part I : Observation of a Tetragonal Distortion of the $\gamma^{\prime}$ Phase at High Temperature by $\gamma$-ray Diffractometry", Phil. Mag. B, 64 (1991), 135-141

14. A.H. Compton and S.K. Allison, "X-rays in Theory and Experiment", Van Nostrand Compagny Inc, Princeton, New Jersey, Toronto and London, p. 718, 1967.

15. D. Bellet and P. Bastie, "Temperature Dependence of the Lattice Parameter of the $\gamma$ and $\gamma^{\prime}$ Phases in the Nickel-Based Superalloy CMSX-2, Part II : Neutron Diffraction Study of the Lattice Parameter Mismatch", Phil. Mag. B, 64 (1991), 143-152

16. J.R. Schneider, "A $\gamma$-ray Diffractometer: A Tool for Investigating Mosaic Structure", J. Appl. Cryst., 7 (1974), 541 546, ibid 7 (1974) 547-554

17. A. Royer et al., "Mesure par Diffraction Neutronique de la Fraction de Phase $\gamma^{\prime}$ dans le Superalliage Monocristallin AMI entre 20 et $1300^{\circ} \mathrm{C}$, Revue de Métallurgie. Science et Génie des 
Matériaux, (1996), in press

18. A. Fredholm, "Monocristaux d'Alliages Base Nickel. Relation entre Composition, Microstructure et Comportement en Fluage à Haute Température", (PhD thesis, Ecole Nationale des Mines de Paris, 1987).

19. M. Veron, Y. Brechet and F. Louchet," Directional Coarsening of $\boldsymbol{\gamma}$ ' Precipitates in Nickel Based Superalloys: Driving Force and Influence of a Prestrain",Phil. Mag. Letters. (1996), in press

20. M. Veron and P. Bastie," Strain Induced Directional Coarsening in Nickel Based Superalloy. Investigation on Kinetics Using SANS Technique", Scripta Metall. Mater., submitted

21. O. Straudo and P. Bastie, private communication with authors, July 1994.

\section{Acknowledgements:}

This work was supported by the French CPR "SSSM" of CNRS. Samples were provided by SNECMA which is gratefully acknowledged for its interest to this study. Experiments were performed at the Institut Laue Langevin at Grenoble (France). We thank its staff and particularly P. Andant and P. Martin from the Iligh Temperature Laboratory and R. Chagnon and P. Ledebt for their help during experiments. We are grateful to D. Bellet from LSP (Grenoble, France), C. Zcyen from ILL and J.L. Strudel from ENSMP ( Evry, France) for fruitful discussions. 